\title{
Archetypes and architecture of time: an artistic inquiry into the nature of time, space and information
}

\author{
Olga Ast \\ Interdisciplinary Conceptual Artist, USA
}

\begin{abstract}
This paper and poster seek to address several fundamental questions about time. What are the natural phenomena and cognitive structures that underlie the human perception of time? What social constructs have evolved around questioning its nature? How did they arise and evolve over the ages? I have been exploring the subject of time merging my background as a conceptual artist with principles of scientific study. My focus has been on the changing visualizations of time through the evolution of human society, from the earliest depictions of the flowing river or the circular uroboros - a snake eating its own tail - to the linear arrow and the paintings of Dali and Magritte, who depict time with modern metaphors of a clock, a train, or the 4th dimension. How have these images influenced scientific, religious and philosophical thought surrounding time? Drawn by now from our collective subconscious, do they naturally bias us towards particular conventional models? And finally, how can an analysis of the visual metaphors of time contribute to the larger dialogue, one that involves scientists, technologists and philosophers, each with their own theories on the subject? This project attempts to answer these questions, and to propose that art is an essential voice in any discussion about time. Can artists and scientists working together bring us closer to an answer to the age-old question - what is time?
\end{abstract}

\section{The problem of time}

I'm a conceptual interdisciplinary artist. One of my goals is to investigate connections between space, time, and information. My work has elements of both scientific and artistic methods and is designed to be a multidisciplinary approach to the problem of time.

Our knowledge of the universe is fragmented. It is assigned to the sciences, mathematics, visual arts, music, literature, religion, philosophy, and so on. All disciplines have developed their own languages with specific terminology. Specialists from different fields understand one another with difficulty, if at all. Yet the universe is not divided into specialized areas of mathematics or physics or poetry. It exists as a whole. I have attempted to avoid specific jargon and inclination toward any cultural or social territories, distance myself from pure artistic or distilled scientific points of view, and reconcile myself to all the imperfections, imprecision, and contradictions of conversational language.

We created a complex artificial environment but still struggle with definition of time and space. One of the main questions about time is probably - what kind of physical phenomena underlie our concept of time passing, if any at all? Since the distant past it has been said and repeated that time may even be an illusion. Is it so? 


\subsection{Time as fear}

The question "What is time?" has been considered to belong to physics or philosophy. To me, it is more psychological: "Why do we experience changes in information as the passage of time?" But what we actually ask is "Why do we age? Why do we not live forever and have to die?" We strive to reverse our decline - go from old age to youth. We are on a never-ending search for the elixir of youth - a cream, a pill, a surgical intervention. We connect the abstract concept of time with our fear of death in our collective consciousness and it transforms into a powerful myth that places an unbearable psychological burden on us. It is one of the prime reasons for our interpretation of informational change as an irreversible passage and decline.
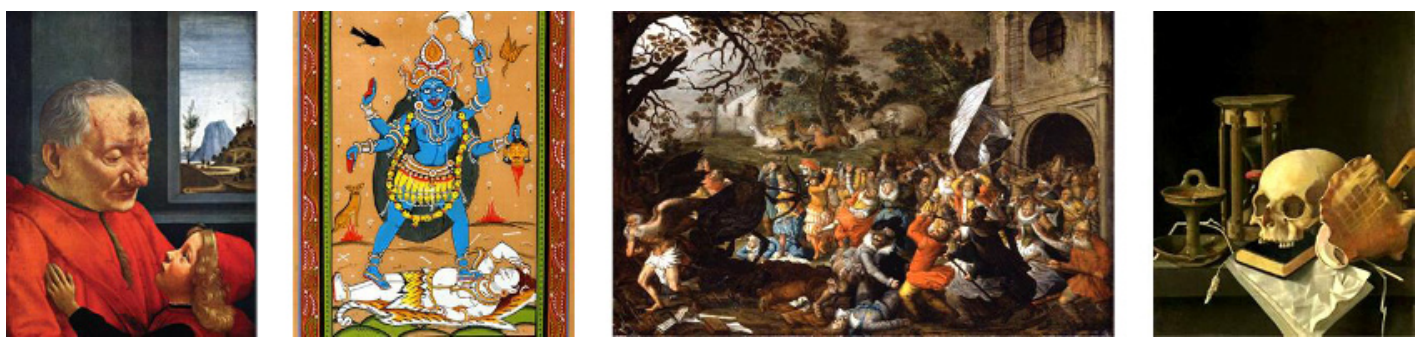

Fig. 1. Time as destruction (from left to right) - 1) An Old Man and His Grandson, c. 1490, by Domenico Ghirlandaio. 2) Kali, warrior goddess of fertility, time, mysteries, destruction and death, Traditional Pata Painting, India. 3) Men and Animals Struggling Against Death and Father Time, c. 1600, by David Vinckboons. 4) Vanitas with Skull and Hourglass, 1686, by Adriaen Coorte.

\section{Visualization of time}

\subsection{Visual metaphors of time}

Visualization is one of the first steps in coming to know an object. What is our mental image or our visual metaphor of time? Frequently, it is the clock - a time-keeping device invented in the middle of the second millennium. The most traditional example is the famous 1931 painting by Salvador Dali entitled The Persistence of Memory with limp clock faces. This demonstrates that even Salvador Dali who proclaimed, "I am Surrealism!" - could not refrain from using the clock metaphor of time.
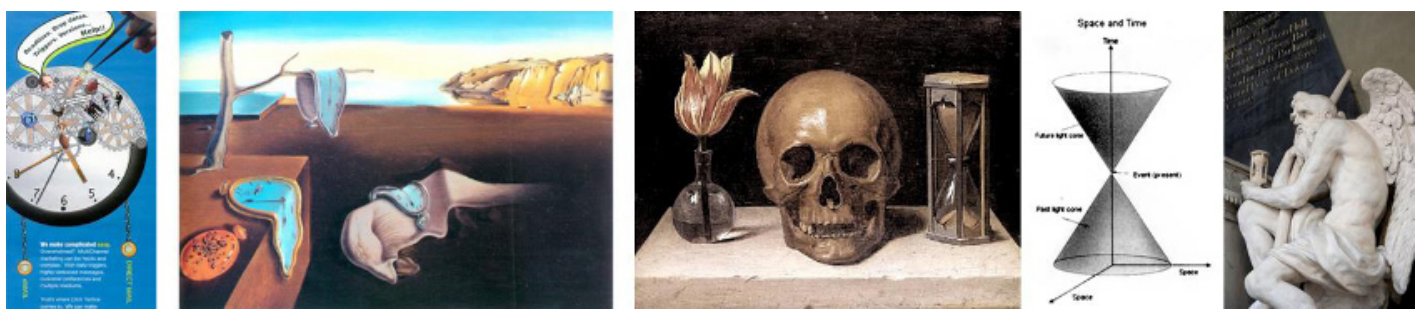

Fig. 2. Time as a clock and as a sandglass (from left to right) - 1) Magazine Advertisement, 2006. 2) The Persistence of Memory, 1931, by Salvador Dali. 3) Vanity, c. 1671, by Philippe de Champaigne. 4) A Brief History of Time, (Fig. 2.4) by Stephen Hawking. 5) Figure of Time, the Henniker tombs, Rochester Cathedral, Kent, England.

Before the mechanical clock and the pendulum we had sundials, sandglasses, water clocks, and candles. Out of all of these, the sandglass (or hourglass) has held the most visual appeal and frequently has been used as a visual metaphor of time and mortality. It shows the separation of time into three distinct chambers or phases. The bottom - the past; the top - the future; and the narrow meetingpoint - the present. The movement of the sand further adds to the metaphoric value. This may also 
remind you of a common contemporary depiction of time that imitates a configuration of sandglass that is filled with an infinite quantity of sand. It is included in A Brief History of Time by Stephen Hawking and other similar books.

But when we speak of the intrinsic or philosophical nature of time, we are met with another set of visual metaphors, not only for how we see time, but also for how we experience it: a river and an arrow. The time-river as immutable, eternal, and existing in constant, flowing motion has proved to be one of the most long-lasting visualizations. The image of a "river of time" has taken on the role of a powerful cross-cultural metaphor, existing in the basic collective memory of the Western world.
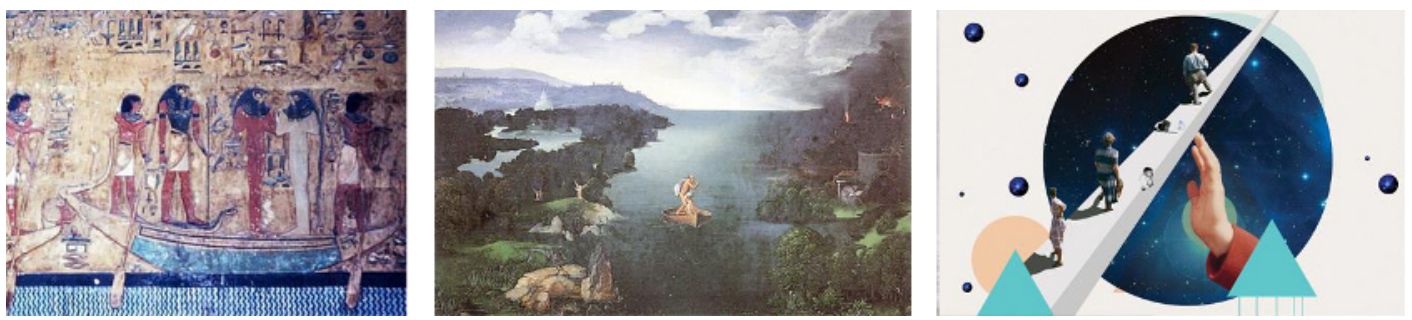

Fig. 3. Time as a river and as an arrow (from left to right) - 1) Wall painting, Tomb of Seti I, Son of Rameses I, 1278 BC, the Valley of the Kings, Egypt. 2) Charon crossing the Styx, 1515-24, by Joachim Patenier. 3) Time's Arrow by Natalie Nicklin. New Scientist Magazine 2011.

The idea of more recent visualization - a so-called "time's arrow" was supposedly first articulated in Europe by Sir Albert Eddington in 1928 in his work Nature of the Physical World. I could not find this analogy prior to that date, nether in The General Theory of Relativity by Albert Einstein nor in similar works on the nature of time, such as the Tertium Organum dedicated to the 4th dimension by the Russian mysticist Pyotr Uspensky. The arrow as our favorite contemporary visual metaphor of time likely came into our collective consciousness alongside the xyz coordinate system. After abstracting the complex spatial environment to three straight lines, we took the next logical step of mapping time along a line. This was an irresistible mathematical temptation. In 1907, mathematician Hermann Minkowski was the first to connect time and the fourth dimension. Twenty years later Sir Eddington articulated the "time's arrow," cementing it not only as an abstract concept, but also as a common visual metaphor. Both metaphors of time as a river and as an arrow represent time as moving in a single, irreversible direction. Just as one cannot enter the same river twice, one cannot retrieve an arrow from its flight.

Looking back at prehistoric symbolism, we find a competing vision of time. Traditional societies largely viewed time as a cyclical process dependent on the eternal repetition of daily, annual, and lifetime patterns. For ancient societies, the future did not hold the unique mystical quality that we attach to it. The future was a symmetrical reflection of both the past and the present. Such belief in the cyclical repetition of patterns was the basis for the Buddhist "wheel of rebirth" and has been preserved in the astrological "wheel of fortune".

One of the notable early symbols that portrays the cycle of time is the Uroboros - a snake eating its own tail. We can trace it back to Neolithic China in 6000 B.C., as well as the Egyptians, the Aztecs, and the Greeks. In 1953, the artist M.C. Escher, known for his complex geometric paradoxes, transformed the Uroboros from its original magical form of a self-devouring snake into a spiral coil that winds around itself in a seemingly endless pattern. It becomes a perfect visual metaphor for any repeated cyclic process. It is the logical continuation of the spiral - a winding circle that collapses in on itself. It seems to have a beginning and an end. And it does not. 

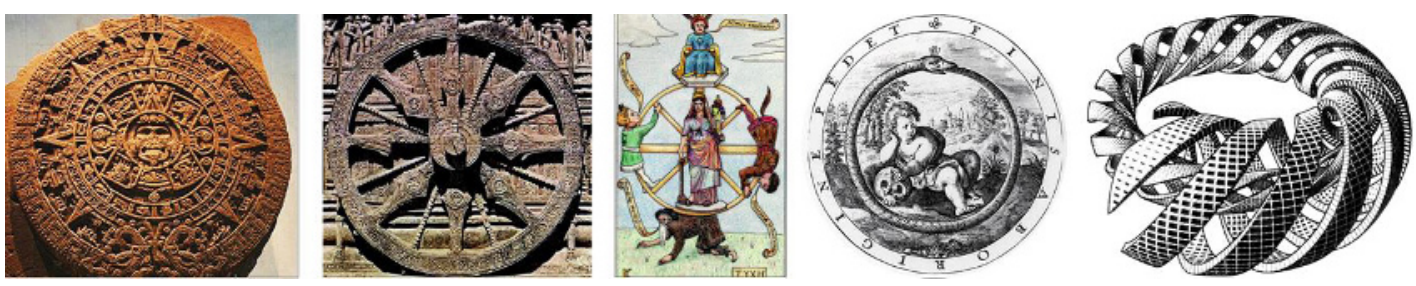

Fig. 4. Time as a circle and as the ouroboros (from left to right) - 1) Aztec Sun StoneCalendar, XV c., Mexico City. 2) Wheel of Salvation, VIII c., India. 3) Wheel of Fortune, XIV c., miniature, France. 4) Uroboros, 1635, G.Wither's collection. 5) Spiral, 1953, by M.C. Escher.

\subsection{Time as trajectory}

Are the changes in objects that we relate to aging and the passage of time as predictable as the circular movements of the clock? The obvious answer is NO. Similar objects can differ in how they change in separate environments, even to the point that we cannot determine their age.

In 1911, the French physicist Paul Langevin proposed the famous "Twin Paradox," based on the work of Albert Einstein. One twin brother goes on a space journey at the speed of light and he will be younger than his twin when he returns. We do not need to go to the extremes of space travel to observe the twin paradox. If you refrigerate a piece of food and leave its twin out on a table you'd prove that the refrigerated food "lives" longer and decays slower. Even temperature can affect the processes of aging. If we can isolate a piece of food in an even colder space, stop all processes of decay, and protect it from all external interactions, we can preserve this piece of food indefinitely.

It means that changes in even identical objects are not actually subject to any unified rate of progress but instead depend on the individual interactions between objects and their environment. The more resistant an object is to change, the longer it can exist in an unchangeable state. In other words, its "life trajectory" is longer than that of an object with a less durable structure. Let's imagine an abstract object so resilient that nothing can actually change it. This object would exist forever. This is where the object's resistance meets time. If time is not an absolute but a relative element of our world then there is no past or future, but only an infinite informational field that is undergoing constant change.

Modern history has been based on a linear understanding of time. But one of the fundamental principles of scientific method is that a hypothesis can be proved only if its conclusion can be repeated in similar conditions. It means that given a set of identical circumstances any observable process will repeat itself in a cyclical fashion. Then any process must have a "life trajectory" that, in ideal settings, can be depicted as a perfect circle.

Outside of tightly controlled experiments, such individual circular trajectories can interact and intersect. We can then suggest that each element has its own life trajectory in our universe and experiences time in its own way. Therefore, time is more accurately visualized not as a river, but as an ocean with competing intersecting currents that move in different directions at varying speed, each following its own cyclical pattern. As human beings with limited life spans, we happen to exist within one local cyclical current, which seems to us to be unidirectional.

\subsection{Time as a mirror}

The most remarkable visualization of human perception of time I have ever seen belongs to René Magritte. His painting "La Réproduction Interdite," (Reproduction Prohibited) portrays a man looking into a mirror and seeing not his face, but his back. Magritte never personally connected this work to time, but it is a perfect example of our perception of it. We look anxiously ahead to the future, but all we can see is the reflection of our past. In this painting, Magritte unwittingly captures the symmetry of time. It is not the simple mirror symmetry that reflects its viewer, but symmetry which, reversed in its own reflection, faces the same direction as the viewer. 

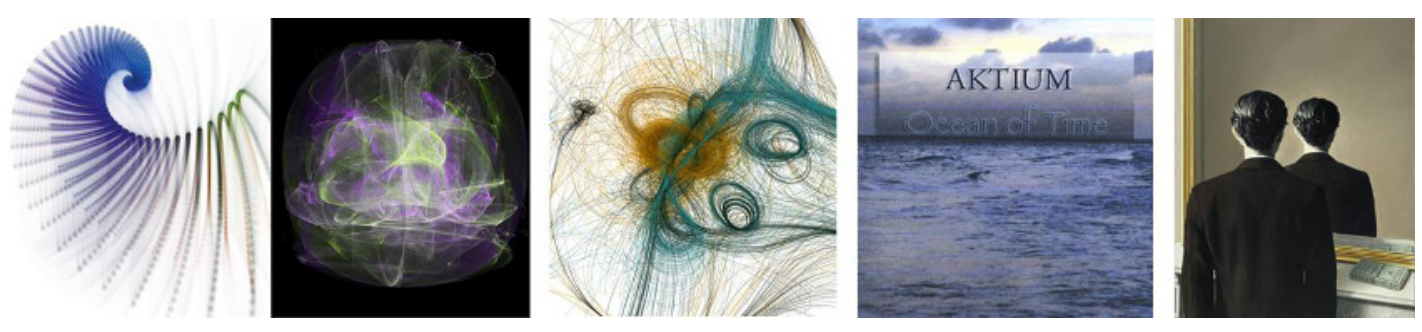

Fig. 5. Time as trajectory, as an ocean and as a mirror (from left to right) - 1) A Virtual Space-Time Travel Machine: an arbitrary surface (Jeener surface 2) in motion, 1993-2006, by Jean-Francois Colonna. 2) Clifford Attractor, 2000-2004, by Paul Richards and Paul Bourke. 3) Path 17, 2001, by Casey Reas (lines in the image reveal paths of autonomous software machines' movement as they respond to stimuli in their environment). 4) Ocean of Time, 2009, Actium Music CD Cover. 5) La Reproduction Interdite, 1937, by René Magritte.

To me this painting looks almost like a formula, like an equation:

the Future equals the Past plus our Desire.

The desire to be in the Future is the other side of our fear of disappearing from there, i.e. our fear of death. This fear pushes us, and transforms into our desire to go into that unknown, to prove to ourselves whether we will exist in the Future. Our desire becomes a driving force that gives the energy to move ahead. This impulse keeps us on the tip of our existence, which we call the Present.

To transform our fear into the desire we have to create a potential difference (like in an electric circuit) between two imaginary points, the Past and the Future. For that we need to overcome our fear of the unknown and attach positive expectations to the future. Our fears and desires are in a constant shaky equilibrium with a little asymmetry. If we cannot attach a positive prediction to the future then a vector of our desire changes its direction from creation to destruction. Therefore we can conclude that our existence in this world happens in the area of the subtle imbalance, that slight asymmetry between the fear of our disappearance and the fear of the future. This asymmetry forces us to go to the Future.

We can even add more equations: the more Desire - the less Fear; more Fear - less Desire to go to our Future. The Fear has to be transformed into Desire to drive us to our existence. But when our future becomes our past we may see it is in fact just a slightly modified reflection of the past.

\subsection{Time as a code}

Our desire transformed from our fear gives us an ability to create subsequent replications in the future. Our body has to replicate its every cell, every molecule, and every atom that it consists of. All these replications are regulated by the code of our DNA. This code developed during the evolution from simple genetic codes in viruses to the more complex codes in humans.

This code exists "between" these replications, and connecting them holds together the bond between past, present, and future copies. I propose that the code that exists between these copies of matter is Time. The code of time is self-developing and evolves from the code that governs replication of viruses into one for mammals or even for social groups and made the evolution of species possible.

It is based in the code that replicates elemental particles. This code and the code's language are selfdeveloping from simple to the complex and are different for different processes. It represents itself differently in the wave-particle manifestation of photons and cyclical planetary motions, frequency of light, and replication of information in human bodies, relationships, and societies.

The code of time is defined by what Magritte captured in his painting, La Reproduction Interdite. The basis of the continuity and rhythm between replications is reflection. His viewer is reflected in a mirror incarnation. Yet his image is reversed in its reflection, facing forward rather than back at him, in order to pass not only his image but also his direction on to the next reflection.

Each replication is a mirror image of the one before, reversed in its own reflection to define not only the replication itself but also a direction of its movement. When we experience what we call a movement through space we actually replicate ourselves through different replications of space. Time 
makes replication possible and holds space together. Time is a primary code for creating the illusions of space and movement. Time is not an illusion, but space and movement are. Time is the code of replication so it can't be touched or sensed; it exists in-between subsequent replications of matter.

\subsection{Time as communication}

The time-code generates and stabilizes the illusion of continuity of space and objects within it. By creating an encoded join between replications, time creates an illusion of movement and an illusion of space, where there is only a series of discrete copies of matter.

Space comes into existence through the code of time. This code - time - is a communication. It is a message from a previous replication to the next. Without this connective, glue-like, communicative tissue, space ceases to exist. Time is a method of constructing and reconstructing space in which we can exist, feel, explore, play games, and invent causalities - crime then punishment.

This code is evolving and plays different games so different types of matter appear - from atoms to planets, from viruses to human beings.

The mysterious Double Slit Experiment (and probably quantum entanglement), which demonstrated that a single photon or electron can pass through two slits simultaneously, seemingly supports this hypothesis that particles also replicate. When an electron passes through the slits, it replicates itself and makes copies, which can coexist in any optimal location in space.

\subsection{Time as shape and as a rhythm}

Our bodies and all objects around us are different forms of response to informational transformation. Therefore, we can say that time forms and molds the shape of physical objects.

Every shape in the universe is a unique record of its time. The form of a particular stone reflects its response to change; the form of a human body similarly reflects both its change along our human evolutionary path and its change during its own existence and experience.

The beating of a drum forms a rhythm out of beats and silences. Matter builds a rhythm out of replications and communicative code in-between that is unique to every entity within it. The existence of the universe may be less mathematical but more musical, like a dance or symphony.

\section{Time Machine}

So, is a Time Machine is possible? Yes. But first, let's ask what we can call a Time Machine.

If time is a code of replication of actualized information then any device that changes that code can be called a Time Machine, We change a code of replication of information with a pen, a computer, a book, a weapon, face creams, etc. Actually everything that we produce changes that code and can be called a Time Machine.

Our body is also equipped with a personal 'time machine' - a brain with the ability to memorize, analyze, and predict. When we can correctly predict something and act accordingly - what is a cause? what is an effect? Of course our prediction can be incorrect, as well as our memory. Our abilities to memorize and predict reflect each other and are symmetrical representations of "past" and "future" that exist simultaneously in our brain, unlike in our physical environment.

But is a Time Travel Machine possible? Can our physical bodies travel to the future or the past? There we are confronted with our wish to make TIME resemble something that we are more familiar with - our SPACE. But if time is a code of replication that is creating the 3D illusion of space then where are we going to travel? Can we travel along the code? Where we are going to travel if there's no the Future, the Past and even the Present?

When we "travel" in space we actually "travel" from one replication of our body to another, which is possible thanks to the code of our DNA that is our personal "time code." Also we "travel" from one replication of space to another. There's no continuity to these replications, they disappear as soon as they appear. It means that it's an illusion of space and movement created by the code of time. 
The time code in our universe has limitations to make this game more interesting, so we cannot change a previous copy. But we create different spaces with different codes. The Internet is also a space with particular programming codes constructing it. The documents and programs that "form" the Internet are not subject to change or aging and can be accessed in different places simultaneously.

The set of natural numbers is also a space. All natural numbers exist here and now; 1 does not exist without 2 or 873, or without infinity. But when we start to count in a precise order from one number to another, e.g. by $\mathrm{N}+1$ (or $\mathrm{N}+3$ ), they suddenly become a subject of "time," a subject to a particular code that allows movement only in one direction, from $\mathrm{N}$ to $\mathrm{N}+1$.

\section{Visualization of Entropy}

Usually the second law of thermodynamics is presented as a proof of the time arrow. We visualize an arrow of time by placing a state of order on one side and direct it to the state of thermodynamic equilibrium or entropy on the other side along a straight line. I'd like to suggest a different possible visual geometrical interpretation of entropy, which is not an attempt to disprove this law.

Let's draw a circle and put the state of thermodynamic equilibrium or entropy at the center. Then we have a visualization that resembles the gravitational force: the state of the equilibrium surrounded by a non-balanced (or non-equilibrium) field of increased complexity states. When something tries to pull away from the original equilibrium then it is pulled back to the center. E.g. as long as our DNA code can pull us to the increased complexity state we can grow and develop. When our resistance and pulling-back force get equalized we can survive, but when our body cannot resist any more it is pulled back to the equilibrium meaning aging and decline.

The real model can be more complex with many levels of possible relative equilibriums, and it may be visualized as in works by British artist Fred Swist.
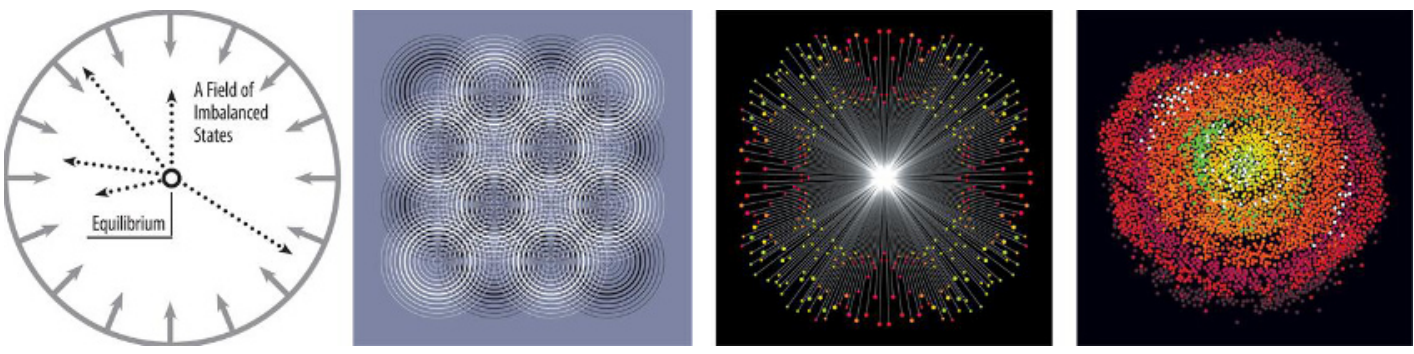

Fig. 6. (from left to right) - 1) Visualization of Entropy by Olga Ast. 2) Three works by

Frédérique Swist: Good vibrations, 2010; Neutrino trails, 2009; Ultracold neutral plasma, 2009.

It seems that a particular equilibrium and surrounding field of possible imbalanced states have to be in somewhat stable relationships. A particular equilibrium probably has to have a radius of influence, where it has the ability to balance everything that does not leave the influence zone. If these relationship become unstable, when something imbalances it too much and goes outside the influence zone then this particular system is destroyed. It's very probable that this is happening now to the Earth's ecology because of the imbalance our high-tech civilization has created. For the last two centuries humans have been almost completely excluded from ecological cycles that nature has been developing over millions of years. Our waste does not fertilize soil anymore; we create garbage and rather than investing in ecological cycles, we destroy them. To continue to exist, everything has to be recycled and reused.

In this visualization of entropy nothing goes from the past to the future in one direction, just back and forth from the equilibrium, which is a center, a start and a finish, alpha and omega of everything. 
